\title{
Über die Einwirkung des Stickoxydes auf einige Metalle bei höherer Temperatur
}

von

\author{
F. Emich.
}

Aus dem chemischen Laboratorium der k. k. technischen Hochschule in Graz.

(Vorgeiegt in der Sitzung am 10. Mai 1894.)

Als ich vor etwa zwei Jahren im Begriffe stand, eine Arbeit über die Einwirkung des Stickoxydes auf einige Metalle abzuschliessen, veröffentlichten Sabatier und Senderens eine Untersuchung ${ }^{1}$ über denselben Gegenstand, deren Ergebnisse nur theilweise mit denen meiner Beobachtungen übereinstimmten. Dadurch war ich veranlasst, damals von der geplanten Publication abzusehen, nicht nur, weil ich zu meiner Beruhigung in einigen Fällen, in welchen die französischen Forscher zu anderen Resultaten gelangt waren, die Experimente unter Anwendung möglichster Vorsicht wiederholen wollte, sondern auch, weil ich mir sagte, dass der Unbetheiligte schwer entscheidet, auf welcher Seite der Irrthum liegt, wenn nur ein Versuch dem anderen gegenübersteht.

Nach dieser Vorbemerkung kann auf den Gegenstand übergegangen werden.

\section{Allgemeiner Theil.}

Mit Rücksicht auf die Thatsache, dass das Stickoxyd zwischen $500^{\circ}$ und $600^{\circ}$ schon eine nachweisbare Zersetzung erleidet, ${ }^{2}$ habe ich, gleich Sabatier und Senderens, die Ein-

1 Compt. rend. 11t, $1429-1432$.

2 Berthelot, Compt. rend., $Z Z, 1448$, und Emich, Monatshefte für Chemie, 13,615 . 
wirkung auf die Metalle in erster Linie bei oder unter der beginnenden Glühhitze studirt; in denjenigen Fällen, in welchen bei diesen Temperaturen keine Anzeichen einer Reaction beobachtet werden konnten, wurde die Wärmezufuhr nach und nach gesteigert, bis volle Rothgluth vorhanden war. Auf die Einhaltung ganz bestimmter Temperaturen habe ich vor dem Erscheinen der erwähnten französischen Arbeit keinen Werth gelegt, weil es doch gewiss - selbst bei der sorgfältigsten Regulirung des Wärmegrades von aussen her - nicht zu vermeiden war, dass die Reactionswärme ganz local eine unberechenbare Temperatursteigerung (die oft ein Erglühen der Metalle hervorrief) zustande brachte. Erst später wurde, um jedem Einwand $z u$ begegnen, bei einzelnen Versuchen das Thermometer $z \mathrm{u}$ Hilfe genommen oder mit Dampfbädern gearbeitet.

Die Metalle gelangten, wie bei Sabatier und Senderens in möglichst fein vertheiltem Zustande zur Anwendung, meist in demjenigen, in welchem man sie bei der Reduction ihrer Oxyde im Wasserstoffstrome gewinnt. Trotz dieser feinen Vertheilung war die Einwirkung des Stickoxydes in der Regel beim ersten Versuch eine unvollständige, d. h. man konnte, einen Fall ausgenommen, durch einmaliges Erhitzen nicht das gesammte Metall ins Endproduct überführen; um zu diesem zu gelangen, musste man vielmehr das zu oxydirende Metall meist wiederholt zerkleinern und neuerdings in der Wärme mit Stickoxyd behandeln. Auf diesen Umstand haben die französischen Forscher anscheinend keine Rücksicht genommen, denn es wäre sonst ganz unerklärlich, dass sich in ihre Arbeit Irrthümer eingeschlichen haben, welche, kurz gesagt, darin bestehen, dass Sabatier und Senderens in einigen Fällen solche Oxydationsstufen als das Endproduct der Einwirkung ansehen, an welche das Stickoxyd noch Sauerstoff abzugeben vermag.

Dadurch wird allerdings das Hauptresultat der in Rede stehenden Arbeit, dass man bei der Einwirkung von Stickoxyd auf Metalle gewöhnlich Oxyde erhalte, welche verschieden sinä von jenen, die an der Luft gebilatet werden - derart beeinflusst, dass es in dieser Form kaum aufrecht erhalten werden 
kann. Nach meinen Versuchen liegt vielmehr der Unterschied zwischen der Wirkung des Sauerstoffes und der des Stickoxydes auf die Metalle in der Regel darin, dass die Oxydation, wenngleich $z$ demselben Endproduct führend, bei Anwendung von Stickoxyd weit langsamer verläuft.

Aus der folgenden Zusammenstellung geht hervor, inwieweit die Resultate der beiderseitigen Arbeiten im Einzelnen von einander abweichen; voranschicken muss ich nur noch, dass Sabatier und Senderens bei Mangan, Wolfram, Uran, Molybdän, Titan, Zinn und Vanadin nicht die Metalle, sondern sauerstoffarme Oxydationsstufen angewandt haben.

Es sind angeführt unter

I... die Oxyde, welche beim Erhitzen mit Sauerstoff entstehen,

II. . . die Oxyde, welche nach Sabatier und Senderens beim Erhitzen mit NO entstehen,

III. . die Oxyde, welche nach meinen Versuchen beim Erhitzen mit NO entstehen.

Ferner bedeutet $» . . . "$, dass die Einwirkung nicht näher studirt wurde, »-- «, dass keine stattfindet.

\begin{tabular}{|c|c|c|c|}
\hline Metall & 1 & II & III \\
\hline $\mathrm{Cu} \ldots . .$. & $\mathrm{CuO}$ & $\mathrm{Cu}_{2} \mathrm{O}$ & $\mathrm{Cu}_{2} \mathrm{O}$ \\
\hline$Z n \ldots \ldots$ & $\mathrm{ZnO}$ & $\ldots$ & $\mathrm{ZnO}$ \\
\hline $\mathrm{Cd} \ldots \ldots$ & $\mathrm{CdO}$ & $\ldots$ & $\mathrm{CdO}$ \\
\hline $\mathrm{Hg} \ldots . .$. & $\mathrm{HgO}$ & - & - \\
\hline $\operatorname{Sn} \ldots \ldots$ & $\mathrm{SnO}_{2}$ & $\mathrm{SnO} \mathrm{O}_{2}$ & $\mathrm{SnO}_{2}$ \\
\hline $\mathrm{Pb} \ldots \ldots$ & $\mathrm{Pb}_{3} \mathrm{O}_{4}$ & $\mathrm{PbO}$ & $\mathrm{PbO}$ \\
\hline $\mathrm{Cr} \ldots \ldots$ & $\mathrm{Cr}_{2} \mathrm{O}_{3}$ & $\cdots$ & $\mathrm{Cr}_{2} \mathrm{O}_{3}$ \\
\hline Mo...... & $\mathrm{MoO}_{3}$ & $\mathrm{MoO}_{2}$ & $\left\{\begin{array}{l}\mathrm{MoO}_{2} \\
\mathrm{Mo}_{3} \mathrm{O}_{12} \\
\mathrm{MoO}_{3}\end{array}\right.$ \\
\hline$W \ldots \ldots$ & $\mathrm{WO}_{3}$ & $\mathrm{~W}_{2} \mathrm{O}_{5}$ & $\mathrm{WO}_{3}$ \\
\hline $\mathrm{U} \ldots \ldots$ & $\mathrm{U}_{3} \mathrm{O}_{8}$ & $\gg \mathrm{U}_{2} \mathrm{O}_{5}$ & $\mathrm{U}_{3} \mathrm{O}_{8}$ \\
\hline Mn...... & $\mathrm{Mn}_{3} \mathrm{O}_{4}$ & $\mathrm{Mn}_{3} \mathrm{O}_{4}{ }^{1}$ & $\mathrm{Mn}_{3} \mathrm{O}_{4}$ \\
\hline
\end{tabular}

1 Die erste Angabe darüber rührt von Guntz her: Bull. d. 1. soc. chimique, 3 e série, t. VII-VIII, No 10, p. 275. 


\begin{tabular}{clcl} 
Metall & \multicolumn{1}{c}{ I } & Il & III \\
$\mathrm{Fe} \ldots \ldots$ & $\mathrm{Fe}_{2} \mathrm{O}_{3}$ & $\mathrm{FeO}$ & $\mathrm{Fe}_{2} \mathrm{O}_{3}$ \\
$\mathrm{Ni} \ldots \ldots$ & $\mathrm{NiO}$ & $\mathrm{NiO}$ & $\mathrm{NiO}$ \\
$\mathrm{Co} \ldots \ldots$ & $\mathrm{Co}_{3} \mathrm{O}_{4}$ & protoxyd & $\mathrm{Co}_{3} \mathrm{O}_{4}$ \\
$\mathrm{Ti} \ldots \ldots$ & $\mathrm{TiO}_{2}$ & $\mathrm{TiO}_{2}$ & $\ldots$ \\
$\mathrm{Vd} \ldots \ldots$ & $\mathrm{VdO}_{2}$ & $\left(\mathrm{Vd}_{2} \mathrm{O}_{3}\right)$ & $\ldots$
\end{tabular}

Demnach liefern von den angeführten sechzehn Metallen nur Kupfer, Blei und Vanadin verschiedene Oxyde, je nachdem man sie im Sauerstoff- oder im Stickoxydstrome erhitzt. Bei Quecksilber liegt der Unterschied darin, dass dasselbe beim Siedepunkte auf Stickoxyd nicht einwirkt, Molybdän endlich bildet das Trioxyd erst bei längerem Glühen im Stickoxydstrome und dann nur wenig davon.

Im Folgenden sollen nur diejenigen meiner Versuche besprochen werden, welche von Sabatier und Senderens entweder nicht oder mit anderem Erfolge ausgeführt worden sind. Mit Rücksicht auf die obwaltenden Umstände wird man entschuldigen, dass hie und da eine etwas grössere Ausführlichkeit bei der Beschreibung Platz gegriffen hat.

\section{Specieller Theil.}

\section{Kupfer}

gibt beim Erhitzen im Stickoxydstrome, wie ich 2 vor dem Erscheinen der Sabatier-Senderens'schen Arbeit angegeben habe, quantitativ Oxydul; zu demselben Resultate sind die französisehen Forscher gelangt. Wir wollen hinzufügen, dass die Einwirkung nicht bloss bei feinvertheiltem, schwammförmigem, sondern auch bei dem als Drahtretz angewandten Metall rasch und volkommen vor sich geht.

\section{Zink,}

das im zusammenhängenden Zustande oder als "Staub* im Stickoxyde erhitzt wird, wirkt erst bei mässiger Rothgluth in bemerkenswerthem Grade auf das Gas ein. Als man das reinste häufliche Metall im Rose'schen Tiegel mit der vollen Gebläseflamme erhitzte, erhielt man ein schön krystallinisches

$1 \mathrm{Vd}_{2} \mathrm{O}_{3}$ veränderte sich beim Erhitzen im $\mathrm{NO}$ nicht.

2 M. $13,70$. 
Product, das nach dem qualitativen Verhalten als Oxyd angesprochen werden musste. Wurde über reinsten Zinkstaub, welcher, in einem Kaliglasrohre auf mässige Rothgluth gebracht, zur Anwendung gelangte, Stickoxyd geleitet, so bildete das austretende Gas an der Luft keine braunen Dämpfe mehr. Das graue Product enthielt eine Spur Stickstoff, ${ }^{1}$ der als Nitrid in demselben vorliegen dürfte (vergl. »Eisen «).

Mit Rücksicht auf diese Versuche ist die Angabe von H. N. Morse und John White, ${ }^{2}$ »das Zink vermag bei starker Hitze Stickoxyd kaum zu reduciren «, entsprechend einzuschränken.

\section{Kadmium}

gab, als Spähne davon in einem Schiffchen im Stickoxydstrome auf beginnende Rothgluth erhitzt wurden, gelbe bis braune Nadeln und schwarzbraune glänzende Körner. Die beiden Producte waren stickstofffrei, lösten sich in Salzsäure ohne Gasentwicklung und ohne Rückstand, die Lösungen gaben die Reactionen der Kadmiumsalze. Es lagen also die beiden Modificationen von Kadmiumoxyd vor, welche z. B. in GmelinKraut's Handbuch ${ }^{3}$ beschrieben werden.

Auch bei Anwendung eines Luftstromes erhält man leicht schön krystallinisches Oxyd, wenn derselbe recht langsam gewählt wird. Im Gegenfalle bedeckt sich das Kadmium mit einer festen Kruste, welche seiner Verdampfung, womit offenbar die Bildung des krystallinischen Productes zusammenhängt, hinderlich ist.

\section{Chrom.}

Das nach Glatzel ${ }^{4}$ bereitete schiefergraue, glanzlose Metallpulver zeigte beim beginnenden Glühen nur Andeutungen

1 Zur Prüfung auf Stickstoff habe ich mit einem Gemenge, das aus der zu untersuchenden Substanz und Kaliumoxalat bestand, die Lassaigne'sche Probe (Erhitzen mit Natrium und Untersuchung auf Cyan) vorgenommen. Die Reaction gelang, was kaum bemerkt zu werden braucht, sehr schön mit allen der Controle halber benützten Stickstoffverbindungen, z. B. mit einer Reihe von Nitraten, einigen Nitriten, Ammonsalzen, mit Magnesiumstickstoff etc.

2 Zeitschr. für anorgan. Chemie, 3, 358.

3 III. Bd., S. 63.

4 B. d. d. ch. G., $23,2127$. 
einer Einwirkung auf das Stickoxyd. Es änderte sein Aussehen nicht und die Gewichtszunahme war gering.

Bei dunkler Rothgluth überzog sich das Chrom mit einer blauen Schichte, deren chemische Natur mit Rücksicht auf die Gegenwart von dem überschüssigen Metall und in Anbetracht der äusserst geringen Menge nicht ermittelt werden konnte.

Bei wiederholtem Erhitzen wurde die Masse, offenbar infolge Bildung von Chromoxyd, grün, doch war selbst durch zehnmaliges Glühen des jedesmal vorher im Achatmörser fein zerriebenen Productes keine Gewichtsconstanz zu erzielen. Die gesammte Gewichtszunahme entsprach nur einem Metallgehalt von $77 \cdot 6 \%$, während die Theorie für das $\mathrm{Oxyd} \mathrm{Cr}_{2} \mathrm{O}_{3}$ $68 \cdot 6 \%$ verlangt. Das Product war stickstofffrei.

1.

2.

3.

4.

5.

Angewandt wurden.. $0 \cdot 4001$

$0 \cdot 4210$

0.4322

0.4385

0.4535

Gewonnen wurden .. 0.4221

$0 \cdot 4336$

$0 \cdot 4404$

$0 \cdot 4557$

0.4617

6.

7.

8.

9.

10.

Angewandt wurden. . $\quad 0 \cdot 4596$

$0 \cdot 4641$

0.4698

$0 \cdot 4733$

$0 \cdot 4840$

Gewonnen wurden . . $\quad 0 \cdot 4666$

$0 \cdot 4713$

$0 \cdot 4743$

0.4854

0.4991

Die geringe Gewichtszunahme beim Erhitzen auf beginnende Rothgluth machte die Annahme möglich, dass das reine Stickoxyd überhaupt nicht auf das Chrom wirke, sondern nur der kleine dissociirte Antheil. Dies scheint jedoch nicht der Fall zu sein, wie ein Versuch lehrt, bei welchem gewogene Mengen des Metalls einmal auf unverändertes, einmal auf theilweise zersetztes Stickoxyd wirkten:

In einem Verbrennungsrohre befanden sich in einer Entfernung von $30 \mathrm{~cm}$ von einander zwei mit Chrom beschickte Schiffchen, die auf ganz dunkle Rothgluth erhitzt wurden, während man das Stück zwisehen ihnen so hell als möglich glühen liess. So kam Chrom Nr. 1 mit frischem, Chrom Nr. 2 aber mit etwas dissociirtem Stickoxyd zusammen. Die Temperatur der Schiffchen wurde selbstverständlich möglichst gleich gehalten, eher dem Chrom Nr. 1 etwas mehr Hitze gegeben.

Chrom Nr. 1 wog $0.6131 \mathrm{~g}$ und nahm um $0.0227 \mathrm{~g}$ oder $3.7 \%$ zu. Chrom Nr. 2 wog $0.5064 \mathrm{~g}$ und nahm um $0.0206 \mathrm{~g}$ oder $4.1 \% \mathrm{zu}$.

Dann wurden die Schiffchen vertauscht und der Versuch wiederholt.

Chrom Nr. 2 (Platz Nr. 1) nahm um 0.0031 g oder $0.6 \%$ zu.

Chrom Nr. 1 (Platz Nr. 2) nahm um $0.0031 \mathrm{~g}^{*}$ oder $0 \cdot 5 \%$ zu. 


\section{Molybdän.}

Zur Darstellung des Metalles wurde käufliches reinstes Molybdänsäureanhydrid im Luftstrome sublimirt, und dann mit Wasserstoff bei nach und nach bis zur Gelbgluth gesteigerten Hitze reducirt. In dem gewonnenen grauen, glanzlosen Product waren die Formen der Molybdänsäurekrystalle noch deutlich erkennbar; es erlitt bei neuerlichem heftigen Glühen im trockenen Wasserstoffstrome keine Gewichtsänderung.

Beim Erhitzen im Stickoxydstrome erhielt ich zuerst eine broncefarbige Masse (Ji- oder Sesquioxyd), später eine blauviolette Verbindung (Dioxyd oder wahrscheinlicher $\mathrm{Mo}_{5} \mathrm{O}_{12}$ ) und schliesslich, als Rothgluth angewandt wurde, eine kleine Menge von Molybdäntrioxyd. - Quantitative Bestimmungen auszuführen war nicht möglich, weil sichtlich stets Gemenge verschiedener Oxyde vorlagen. Man hatte also zur Feststellung der chemischen Natur nur einen zum Theil unsicheren Anhaltspunkt: die Farbe, welche zufolge der vorliegenden Angaben 1 bei den niederen Oxyden je nach den Gewinnungsmethoden eine verschiedene sein kann.

Sabatier und Senderens haben beim Erhitzen von Molybdänsesquioxyd im Stickoxydstrom Erglühen und Bildung einer violetten Verbindung, welche sie für Dioxyd erklären, beobachtet.

\section{Wolfram,}

aus sorgfältig gereinigtem Trioxyd wie Molybdän bereitet, erglühte im gepulverten Zustande, wenn es im Stickoxydstrome auf etwa $500^{\circ}$ erhitzt wurde und bildete unter beträchtlicher Anschwellung traubige Massen, die zweifellos nicht einheitlicher Natur waren, indem einzelne Partien gelb, andere braun, wieder andere grün bis blau erschienen.

Nach viermaligem Zerreiben und Glühen im Gasstrome betrug, nachdem das Product rein gelb geworden und Gewichtsconstanz eingetreten war, der Metallgehalt $79 \cdot 28 \%$, es lag also Trioxyd vor, welches $79 \cdot 31 \%$ Wolfram enthält.

1.

2.

3.

4.

$\begin{array}{lllll}\text { Angewandt...... } & 0.6926 & 0.4730 & 0.4753 & 0.4750 \mathrm{~g} \\ \text { Gewonnen ..... } & 0.8590 & 0.4782 & 0.4780 & 0.4751 \mathrm{~g}\end{array}$

Das Product der ersten Einwirkung wurde mit negativem Erfolge auf Stickstoff geprüft.

1 Vergl. z. B. D ammer's neues Handbuch der anorg. Ch., III, 592. 
Sabatier und Senderens haben aus Wolframdioxyd unter Erglühen blaues »Pentoxyd « erhalten.

\section{Uran.}

Die Darstellung des Metalles erfolgte nach PeligotZimmermann ${ }^{1}$ durch Reduction von Tetrachlorid mit Natrium in einer verschraubten Stahlbombe.

Das Product, ein feines, schwarzes Pulver, war nicht ganz oxydfrei, denn $0 \cdot 3888 g$ nahmen beim Glühen an der Luft $0 \cdot 0651 g$ auf, woraus sich für das entstandene Uranuranat $85 \cdot 6^{\circ} \%$ Metall statt $84.9 \%$ berechnen würden; für meine Zwecke war dieser Umstand belanglos.

Das Metall verbrannte im Stickoxydgas äusserst leicht und mit fast blendender Lichterscheinung; nach dreimaligem Erhitzen war nahezu Gewichtsconstanz eingetreten:

1.

2.

3.

$$
\begin{array}{llll}
\text { Angewandt..... } & 0 \cdot 3790 & 0 \cdot 4314 & 0 \cdot 4351 \mathrm{~g} \\
\text { Gewonnen ..... } & 0 \cdot 4368 & 0 \cdot 4377 & 0 \cdot 4357 \mathrm{~g}
\end{array}
$$

Das Product enthielt also $85 \cdot 4 \%$ Metall, es war stickstofffrei (was mit Rücksicht auf die von Uhrlaub behauptete, von Hillebrand ${ }^{2}$ jedoch widerlegte Existenz eines Oxynitrides $\mathrm{U}_{10} \mathrm{~N}_{4} \mathrm{O}_{18}$ erwähnt werden mag). Beim Glühen im Sauerstoffe änderte es sein Gewicht nicht wesentlich.

$0.5675 \mathrm{~g}$ (von anderer Darstellung, wobei nach viermaligem Erhitzen Gewichtsconstanz eingetreten war) gaben $0.5667 \mathrm{~g}$.

Es lag also Uranuranat $\mathrm{U}_{3} \mathrm{O}_{8}$ vor.

Sabatier und Senderens geben an, beim Erhitzen von Uranoxydul $\mathrm{UO}_{2}$ unter $500^{\circ}$ im Stickoxydstrom die schwarze Verbindung $\mathrm{U}_{2} \mathrm{O}_{5}$, erhalten zu haben.

Hiezu muss zunächst bemerkt werden, dass nach Cl. Zimmermann's äusserst sorgfältigen und ausführlichen Untersuchungen ${ }^{3}$ die "Verbindung $\mathrm{U}_{2} \mathrm{O}_{j}$ von Peligot kein einheitlicher Körper ist.

1 B. B. 15,840 .

2 Zeitschr. für anorgan. Ch., 3, 241.

3 A. d. Ch., 232, $273 \mathrm{ff}$. 
Trotzdem habe ich Versuche mit Uranoxydul nach dem Erscheinen der französischen Arbeit aufgenommen; es ist mir aber nicht gelungen, etwa durch mehrtägiges Erhitzen von Uranoxydul mit Stickoxyd auf $360^{\circ}$ jenem die einem Oxyd $\mathrm{U}_{2} \mathrm{O}_{5}$ entsprechende Sauerstoffmenge zu appliciren.

Der Versuch wurde folgendermassen angestellt: $1 g$ Uranoxydul, gewonnen durch Reduction von Uranuranat mit Wasserstoff bis zur Gewichtsconstanz, wurde in ein Einschmelzrohr von circa $120 \mathrm{~cm}^{3}$ Inhalt gebracht. Dasselbe wurde ausgepumpt, dann mit Stickstoff gefüllt, dieser durch Stickoxyd verdrängt, später zugeschmolzen und endlich 70 Stunden ununterbrochen im Quecksilberdampfbad erhitzt. Vom gebildeten Product nahmen $1.0318 \mathrm{~g}$ beim Glühen an der Luft um $0.0215 \mathrm{~g}$ oder $2 \cdot 09 \% \mathrm{zu}$, ein Oxyd von der Formel $\mathrm{U}_{2} \mathrm{O}_{5}$ hätte nur $0.95 \%$ aufnehmen dürfen. Ebensowenig habe ich beim Leiten von Stickoxyd über Uranoxydul, das im Schiffchen im Verbrennungsrohr bis fast zum Glühen erhitzt wurde, ein solches Oxyd gewinnen können.

Berücksichtigt man noch, dass Sabati er und Senderens analytische Daten über das angebliche $\mathrm{U}_{2} \mathrm{O}_{5}$ nicht mittheilen, sondern höchstwahrscheinlich die Natur des Productes nur aus der schwarzen Farbe (die aber nach Cl. Zimmermann unter Umständen auch dem Oxyd $\mathrm{U}_{3} \mathrm{O}_{8}$ zukommt) erschlossen haben, so wird man nicht fehlgehen, wenn man die Angabe der genannten Autoren als eine irrthümliche bezeichnet.

\section{Eisen.}

Sabatier und Senderens geben an, dass sich schwammförmiges Eisen bei $200^{\circ}$ im Stickoxydstrome entzünde, das Product sei "protoxyd gris noir ", in welchem $77.0 \%$ Metall gefunden werden, die Rechnung verlange auch $77 \cdot 0 \%$ Fe.

An dieser Angabe ist zunächst ein Versehen zu berichtigen, denn ein Oxyd mit $77.0 \%$ Eisen existirt nicht; Eisenoxydul, das allein gemeint sein kann, enthielte $77 \cdot 78 \%$ Metall.

Ich kann bestätigen, dass die Entzündung von fein vertheiltem Eisen im Stickoxyde sehr leicht erfolgt (eine Temperaturmessung habe ich hier so wenig wie in anderen Fällen vorgenommen, weil der Entzündungspunkt sicher von der Fein- 
heit des Materials abhängt und constante Resultate kaum erzielbar sein dürften).

Bei den ersten Versuchen $(A)$ wurde Eisen im Schiffchen im Verbrennungsrohre erhitzt und nach dem Verglimmen noch etwa drei Viertelstunden auf beginnender Rothgluth erhalten. Nach dem Erkalten entfernte man (wie in allen Fällen, wo nichts Näheres angegeben ist) das im Rohre befindliche Stickoxyd durch schnelles Evacuiren und zerrieb den Inhalt des Schiffchens. Das Product hatte etwa die Farbe des gepulverten Rotheisensteins, gab aber an den Magnet noch viel ab. Nach zweimaliger weiterer Oxydation nahm es bei neuerlichem Erhitzen nur mehr um $0.2 \mathrm{mg}$ zu und enthielt $70.3 \%$ Eisen.

$0.1713 g$ gaben nach der Reduction mit Wasserstoff $0 \cdot 1205 g$.

Es lag also Eisenoxyd (das $70 \cdot 0 \%$ Fe enthält) vor.

Nach dem Erscheinen von Sabatier und Senderens' Abhandlung habe ich versucht, durch Anwendung eines geringeren Hitzegrades das erwähnte schwarzgraue Eisenoxydul zu gewinnen. Es ist mir aber weder bei $360^{\circ}$ noch bei $450^{\circ}$ gelungen, einen einheitlichen Körper von der durch die Formel $\mathrm{FeO}$ ausgedrückten Zusammensetzung zu erhalten. Wir wollen zunächst einen Versuch genauer beschreiben, bei welchem die Einwirkung bei der Temperatur des siedenden Schwefels stattfand.

Versuch $B$. In ein zum vierten Theile mit Schwefel gefülltes Kölbchen wurde eine Eprouvette eingesetzt, in welche eine zweite gewogene eingefügt werden konnte. Die letztere beschickte man mit $1.5858 g$ Eisenschwamm und adjustirte sie im übrigen derart, dass sich ein beliebiger Gasstrom ins Innere leiten liess. Nun wurde die darin befindliche Luft durch reinen Stickstoff, dieser dann durch Stickoxyd verdrängt, ferner der Schwefel zum Sieden erhitzt und der Apparat drei Viertelstunden in Thätigkeit gelassen; schliesslich musste das Product im Stickstoffstrome erkalten.

Nach dem Auseinandernehmen des Apparates wog das oxydirte Metall $1.8115 g$, die Einwirkung hatte aber sichtlich nur einen Theil des Eisens betroffen. Das Product war grauschwarz; es wurde nach dem Zerreiben vom Magnet stark und 
bis auf das letzte Theilchen angezogen, enthielt also offenbar noch kein Eisenoxyd oder nur sehr wenig davon. Man trocknete das Pulver (wie bei ähnlichen Versuchen stets! - weil während des Zerreibens immer etwas Feuchtigkeit aufgenommen wurde) im Stickstoffstrome bei etwa $250^{\circ}$ und behandelte es hierauf wie oben wieder mit Stickoxyd, wobei $1 \cdot 8064-1 \cdot 9476 g$ gaben. Beim neuerlichen Zerreiben kamen noch unangegriffene Metallflitter zum Vorscheine und nach dem Behandeln mit dem Magnet blieb eine kleine Menge ( $2 \mathrm{mg}$ ) eines rothes Pulvers übrig. Nach der dritten Behandlung $(1.9289 g$ gaben dabei $1 \cdot 9781 \mathrm{~g})$ hatte das Präparat einen rothbraunen Schimmer und lieferte $80 \mathrm{mg}$ rothes Pulver. (Das Product hatte jetzt ganz annähernd den Eisengehalt des Oxyduls, nämlich $79 \cdot 7 \%$.)

Nachdem die geschilderten Operationen noch zweimal wiederholt worden waren, hatte man etwa ein Viertel Gramm einer Substanz erhalten, welche vom Magnet kaum angezogen wurde und bei neuerlichem Erhitzen auf $450^{\circ}$ im Stickoxyd keine beträchtliche Gewichtszunahme erlitt: $0.2371 \mathrm{~g}$ gaben $0.2386 \mathrm{~g}$. - Bei der Reduction mit Wasserstoff erhielt man aus $0.2320 \mathrm{~g}$ Substanz $0.1649 \mathrm{~g}$ Eisen oder $71 \cdot 1 \%$. Es lag also zwar noch kein reines Eisenoxyd (welches $70.0 \%$ Fe enthält) vor; wenn man aber bedenkt, dass der Körper, wie gesagt, von rother Farbe war, und dass Eisenoxyduloxyd schon $72 \cdot 4^{\%} \%$ Metall enthält, so wird man über die Natur des bei genügend langer Einwirkung zu erwartenden Körpers nicht Zweifel hegen.

Bei einem analog unter Anwendung von Quecksilberdampf angestellten Versuch erhielt man nach der vierten Oxydation eine Spur des rothbraunen Pulvers.

Als einen weiteren Versuch dieser Art führe ich noch den folgenden an.

Versuch $C$. Eine kleine Menge mit Wasserstoff reducirten Eisens wurde in ein $U$-förmig gebogenes Einschmelzrohr gebracht, welches man vor dem Schliessen erst mit Stickstoff, dann, um diesen zu verdrängen, mit Stickoxyd füllte. Der Inhalt des Rohres war derart bemessen, dass das Stickoxyd nöthigenfalls das ganze Eisen in Oxyd hätte überführen können. Das Rohr erhitzte man acht Stunden lang im Schwefeldampf und 
verdrängte hierauf das darin befindliche Gas wieder durch Stickstoff. Das zusammengebackene oxydirte Metall lieferte beim Zerreiben im Achatmörser ausser Flittern von unangegriffenem Eisen eine verhältnissmässig gar nicht unbedeutende Menge eines rothbraunen Pulvers, offenbar Eisenoxyd.

Es wurde weiters untersucht, ob Eisenoxyduloxyd in der Hitze auf Stickoxyd einwirkt und gefunden, dass dies ganz zweifellos der Fall ist. Die Einzelnheiten des Versuches waren die des unter $B$ besprochenen, mit dem Unterschiede, dass sich: das Eisenoxyduloxyd neben einem Thermometer im Porzellanschiffchen im Verbrennungsrohr befand.

Versuch $D .0 \cdot 9619 \mathrm{~g}$ Eisenoxyduloxyd wurden im Stickstoffstrome auf $380^{\circ}$ erhitzt, der Stickstoff wurde durch Stickoxyd verdrängt und nach drei Viertelstunden wieder Stickstoff eingeleitet, während der Apparat abkühlte. Das Präparat war rothbraun geworden und hatte um $0.0271 \mathrm{~g}$ an Gewicht zugenommen. - Bei einem anderen Versuch zeigten $0 \cdot 2318 g$ eine Gewichtsvermehrung um $0.0079 \mathrm{~g}$.

Darnach kann mit Sicherheit behauptet werden, dass das Eisenoxyd das Endproduct der Einwirkung von Eisen auf Stickoxyd darstellt.

Bemerkenswerth ist noch, dass das mit Sticlioxyd oxydirte Eisen stets geringe Mengen von Stickstoff enthält, welche u. A. leicht nachgewiesen werden können, wenn man die Probe in einem verschliessbaren Gläschen mit Wasser anrührt und einen rothen Lakmuspapierstreifen darüber gibt; derselbe wird nach einiger Zeit stets blau.

In welcher Form ist der Stickstoff in dem Producte enthalten?

Die Analyse kann auf diese Frage keine Antwort geben schon desshalb nicht, weil die Stickstoffmengen sehr klein sind. In zwei Fällen betrugen sie 0.1 und $0.05 \%$.

1. $1.2297 \mathrm{~g}$ Substanz gaben, mit Lauge gekocht, Ammoniak, welches $0.25 \mathrm{~cm}^{3}$ Salzsäure $\left(1 \mathrm{~cm}^{3}=5.45 m g \mathrm{NH}_{3}\right)$ neutralisirte. Das Präparat war durch einmaliges Überleiten von Stickoxyd über Eisenschwamm erhalten worden, es enthielt $84.1 \%$ Eisen $(0.7707 \mathrm{~g}$ gaben $0.9261 \mathrm{~g}$ Eisenoxyd).

2. $2 \cdot 9775 g$ Substanz lieferten Ammoniak, das $0.35 g$ der obigen Säure neutralisirte; das wie oben gewonnene Präparat enthielt $82.4 \%$ Eisen $(0 \cdot 6168 \mathrm{~g}$ gaben $0.7261 \mathrm{~g}$ Oxyd). 
Es bleibt also nur zu überlegen, ob unter den gegebenen Umständen die Entstehung einer Eisen-Stickstoff-Sauerstoffoder die einer Eisen-Stickstoff-Verbindung mehr Wahrscheinlichkeit für sich hat. Wenn man bedenkt, dass die bekannten Eisensalze der Salpetersäure in höherer Temperatur nicht sehr beständig sind, wenn man dem entgegenhält, dass der Eisenstickstoff nach Rogstadius ${ }^{1}$ bei Gegenwart von überschüssigem Eisen Weissgluth verträgt, und nach Briegleb und Geuthe ${ }^{2}$ direct aus seinen Bestandtheilen entstehen kann, wenn man endlich berücksichtigt, dass bei der Einwirkung von Eisen auf Stickoxyd nascirender Stickstoff vorhanden ist, so wird man zur Annahme gedrängt, dass der Stickstoffgehalt des Oxydationsproductes durch kleine Mengen von Eisennitrid bedingt wird. Damit steht in Einklang, dass das aus Eisenoxyduloxyd und Stickoxyd entstehende Product stickstofffrei ist.

Ich habe mich vergebens bemüht, den Versuch - Erhitzen von Eisen im Stickoxydstrome - derart anzuordnen, dass, wenn auch nur für kurze Zeit, sichtlich ein völliger Verbrauch des Stickoxydes eingetreten wäre: man kann stark oder schwach erhitzen, viel oder wenig Eisen nehmen, den Gasstrom schnell oder langsam wählen, es gelingt nicht, eine vorgelegte Sperrflüssigkeit zum Zurüclisteigen zu bringen.

\section{Kobalt.}

Sabatier und Senderens geben an, dass sich schwammförmiges Kobalt bei $150^{\circ}$ im Stickoxydstrome entzünde und braunes "protoxyd " bilde; eine Analyse wird nicht mitgetheilt.

Ich hatte vor dem Erscheinen der vielerwähnten Arbeit ebenfalls schon beobachtet, dass die Entzündung vor dem Beginne des Glühens erfolgte. Das Product war schwarz, vielleicht mit einem Stich ins Grüne, doch ist dieser Umstand mit Rücksicht auf die verschiedenen Färbungen, welche viele Oxyde je nach dem Zustande ihrer Vertheilung zeigen, kaum von Belang. Das einmal oxydirte Metall gab mit Salzsäure noch Wasserstoff; es wurde, wie dies beim Eisen (Versuch $A$ ) beschrieben

1 Gmelin-Kratt's Handb., III, 365.

2 Ann. d. Chemie, $123,238$. 
ist, so lange abwechselnd gepulvert und erhitzt, bis die Gewichtszunahme einige Zehntelmilligramme betrug. Ich führe von einem Versuch die Daten an.

1. $2.3 . \quad 3$.

Angewandt $\ldots \ldots \ldots \cdot 0.3638 \quad 0.4809 \quad 0.4835 \quad 0.4821 g$

Gewonnen ...... $0.4831 \quad 0.4851 \quad 0.4840 \quad 0.4824 g$

Die totale Gewichtszunahme entsprach einem Metallgehalt von $74.5 \%$ für das Endproduct.

In einem anderen Falle enthielt dasselbe nach siebenmaligem Erhitzen $73 \cdot 9 \%$. Diese Zahlen sprechen für die Bildung von $\mathrm{Oxyduloxyd} \mathrm{Co}_{3} \mathrm{O}_{4}$, welches $73 \cdot 44 \%$ Kobalt enthält. Die Producte entwickelten mit Salzsäure Chlor.

So weit war meine Untersuchung abgeschlossen, als die Arbeit der französischen Forscher erschien und in mir Zweifel erweckte, ob ich die Oxydation doch nicht durch Anwendung einer zu hohen Temperatur - ich hatte bei Dunkelrothgluth gearbeitet - zu weit getrieben hätte.

Die Versuche wurden daher neuerdings aufgenommen, dabei aber nicht vom Metall, sondern vom Kobaltoxydul ausgegangen, in der Voraussetzung, dass dieses beim Erhitzen im Stickoxydstrome an Gewicht nicht zunehmen würde, wenn es selbst das Einwirkungsproduct von Stickoxyd auf Kobalt darstellt, oder wenn diese Rolle einem noch sauerstoffärmeren Oxyde zufiele.

Behufs Gewinnung von Kobaltoxydul fällte man Kobaltnitrat mit Kaliumcarbonat, trocknete den vollkommen ausgewaschenen Niederschlag nach Entfernung der obersten Partien, welche sich anscheinend etwas oxydirt hatten und glühte ihn im Stickstoffstrome. Das Product war ein grüngraues Pulver, welches mit Salzsäure keine nachweisbaren Mengen von Chlor entwickelte und $78 \cdot 63 \%$ Metall enthielt.

$1.2770 \mathrm{~g}$ gaben bei der Reduction mit Wasserstoff $1.0042 \mathrm{~g}$ Metall. Die Rechnung verlangt für $\mathrm{CoO} 78 \cdot 67 \%$.

Das Kobaltoxydul wurde bei einer Reihe von Versuchen genau wie das Eisenoxyduloxyd (Versuch $D$ ) im Stickoxydstrome erhitzt. Die Producte waren dunkelgrau bis schwarz, 
entwickelten mit Salzsäure Chlor und enthielten keinen Stickstoff (wovon man sich nicht nur in der beim Zink angegebenen Weise, sondern in einem Falle überdies durch einen nach Art der Dumas'schen Bestimmung angeordneten Versuch überzeugte).

In der folgenden Übersicht sind die Einzelheiten der Versuche zusammengestellt.

\begin{tabular}{cccccccc} 
Versuch & $\begin{array}{c}\text { Angewandte } \\
\text { Oxydu1- } \\
\text { menge }\end{array}$ & $\begin{array}{c}\text { Temp. } \\
\text { (uncorr.) }\end{array}$ & Dauer & $\begin{array}{c}\text { Gewicht nach } \\
\text { NO-Ein- } \\
\text { wirkung }\end{array}$ & \multicolumn{2}{c}{ Zunahme } \\
I & $0 \cdot 4964 g$ & $400^{\circ}$ & $1^{\text {h }}$ & $0 \cdot 5047 g$ & $0 \cdot 0083$ & $1 \cdot 7$ \\
II & $0 \cdot 2590$ & 400 & 1 & $0 \cdot 2685$ & $0 \cdot 0095$ & $3 \cdot 7$ \\
III & $0 \cdot 1900$ & 404 & $3 / 4$ & $0 \cdot 1941$ & $0 \cdot 0041$ & $2 \cdot 1$ \\
IV & $0 \cdot 2907$ & 401 & 2 & $0 \cdot 3070$ & $0 \cdot 0163$ & $5 \cdot 7$ \\
V & $1 \cdot 2770$ & 396 & $1^{3} / 4$ & $1 \cdot 3210$ & $0 \cdot 0440$ & $3 \cdot 2$
\end{tabular}

Da mithin zweifelsohne das Stickoxyd auf Kobaltoxydul in bemerkenswerthem Grade einwirkt, kann dieses unmöglich das Endproduct der Reaction sein; als solches ist vielmehr ein sauerstoffreicheres $\mathrm{Oxyd}$, nach dem früher Angegebenen höchstwahrscheinlich das Kobaltoxyduloxyd zu bezeichnen.

Wenn auch die Reihe der untersuchten Metalle keine grosse ist, so würde sie immerhin zur Aufstellung einiger allgemeiner Gesichtspunkte genügen; ich gedenke auf dieselben zurückzukommen, wenn einmal das Verhalten der Nichtmetalle zu Stickoxyd genau studirt ist.

Es möge gestattet sein, anhangsweise noch einen anderen Gegenstand zu berühren.

Im Jahre 1892 habe ich ein par Versuche beschrieben, ${ }^{1}$ aus denen hervorging, dass Stickoxyd und Sauerstoff, welche behufs vollkommener Trocknung mit Phosphorpentoxyd in Röhren eingeschlossen waren, bei ihrem Zusammentreffen unter Bildung brauner Dämpfe reagirten. Seither ist eine

1 Monatshefte für Chemie, 13, 86. 
vorläufige Mittheilung von Baker ${ }^{1}$ erschienen, nach welcher die beiden Gase im vollkommen trockenen Zustande nicht auf einander wirken.

Es liegt mir selbstverständlich vollkommen ferne, die Resultate anzuzweifeln, welche der auf diesem Gebiete hochverdiente englische Forscher veröffentlicht hat, ich möchte nur bemerken, dass ich seit dem Erscheinen der Baker'schen Arbeit, die seinerzeit von mir beschriebenen Versuche verschieden abgeändert wiederholt, die beiden Gase bis zu drei Vierteljahren mit Phosphorpentoxyd (auch mit Schwefelsäure) getrocknet und trotzdem stets sofortige Bräunung beobachtet habe, wenn die das eine Gas vom anderen trennende Scheidewand durchstossen wurde. Es scheint also, dass für meine Versuchsanordnung wirklich der Einwand Traube's ${ }^{2}$ gilt, d. h. dass die Phosphorsäure die Rolle des Sauerstoffüberträgers, welche sonst dem Wasser zukommt, übernommen hat.

1 Chem. News, 67, 263.

2 Berl. Ber., 26, 1475. 